\title{
latrogenic Perforation of Upper Pouch in Pure Esophageal Atresia: A Rare Complication and Review of Literature
}

\author{
Sandesh Parelkar ${ }^{1}$ Dinesh Mundada ${ }^{1}$ Prashant Joshi ${ }^{1} \quad$ Beejal Sanghvi ${ }^{1} \quad$ Satish Kapadnis ${ }^{1}$ Sanjay Oak ${ }^{1}$ \\ ${ }^{1}$ Department of Paediatric Surgery, Seth GS Medical College and King \\ Edward VII Memorial Hospital, Mumbai, Maharashtra, India \\ Address for correspondence Prashant Joshi, MS, MCh, FMAPS, Assistant \\ Professor, Department of Paediatric Surgery, Seth GS Medical College and \\ King Edward VII Memorial Hospital, Acharya Dhonde Marg Parel, Mumbai, \\ Eur J Pediatr Surg Rep 2013;1:21-23. \\ Maharashtra 400012, India (e-mail: pbjoshiisat@gmail.com).
}
Abstract
Keywords
- esophageal atresia
- upper pouch
- perforation
- iatrogenic

latrogenic perforation of the neonate's pharynx and esophagus with normal anatomy was first described by Eklöf et al in 1968. It typically occurs in severely premature neonates who have undergone repeated traumatic attempts at endotracheal intubation or passage of orogastric tubes. It may also mimic esophageal atresia (EA). Perforation of upper pouch in tracheoesophageal fistula with EA was rarely reported. We report a $1,400 \mathrm{~g}$ (32 weeks) neonate with pure EA and iatrogenic perforation of upper pouch due to use of catheter for diagnostic radiography.

\section{Introduction}

Pharyngeal and esophageal perforation secondary to nasogastric (NG) or orogastric (OG) tube placement is rare and occurs most frequently in preterm infants who have weaker pharyngeal muscular structures and are at increased risk of iatrogenic esophageal perforation. ${ }^{1,2}$ Esophageal perforation in a fullterm infant may occur with repeated attempts to place NG, OG, or endotracheal tubes. Eklöf et al first described iatrogenic esophageal perforation in $1968 .^{3}$ Review of the literature reveals misdiagnosed esophageal atresia (EA) in the setting of esophageal perforation. Some infants have mistakenly undergone unnecessary thoracotomy for the same. ${ }^{1,4}$ However, esophageal perforation with EA is exceedingly rare. Perforation of the upper pouch in tracheoesophageal fistula (TEF) with EA was reported by Wright and Noblett, almost 35 years ago (1978). ${ }^{5}$ Here, we report a case of pure EA that had this rare complication of perforation of blind upper pouch of esophagus.

\section{Case Report}

A newborn baby weighing $1,400 \mathrm{~g}$ was transferred to our neonatal intensive care unit on day 3 after birth with symptoms of frothing after birth, with suspected EA. The baby was born at 32 weeks' gestation by normal vaginal delivery to a 22 -year-old gravida 1 , para 1 mother who went in premature

received

December 13, 2012

accepted after revision

January 2, 2013

published online

March 20, 2013

labor. She had a normal prenatal ultrasound scan. She was hemodynamically unstable and required vasopressors. Baseline hematological investigations were normal except $C$ reactive protein, which was 31 ( $N=0-6 \mathrm{mg} / \mathrm{L})$. To our surprise, the stiff rubber catheter (10 fr), passed to document the atresia and for taking the X-ray, went smoothly and effortlessly for $25 \mathrm{~cm}$.

But, the X-ray showed the tube ending in the right pleural cavity with right pneumothorax and gasless abdomen suggestive of pure EA with possible iatrogenic perforation of the upper pouch (-Fig. 1). The catheter was removed immediately. Owing to increasing respiratory distress, the baby was intubated and ventilator support was provided. Right-sided intercostal drainage tube was inserted. The patient gradually improved and stabilized with ventilatory support.

Cervical esophagostomy and feeding gastrostomy without thoracotomy were done. There was $0.5 \times 0.3 \mathrm{~cm}$ perforation at the tip of the pouch with irregular margins ( - Fig. 2). Left cervical esophagostomy was done after mobilizing upper pouch completely.

\section{Discussion}

Pharyngeal and esophageal perforations are rare but wellknown complications of NG and OG catheter placement.

(c) 2013 Georg Thieme Verlag KG Stuttgart · New York
DOI http://dx.doi.org/ $10.1055 / \mathrm{s}-0033-1341417$. ISSN 2194-7619. 


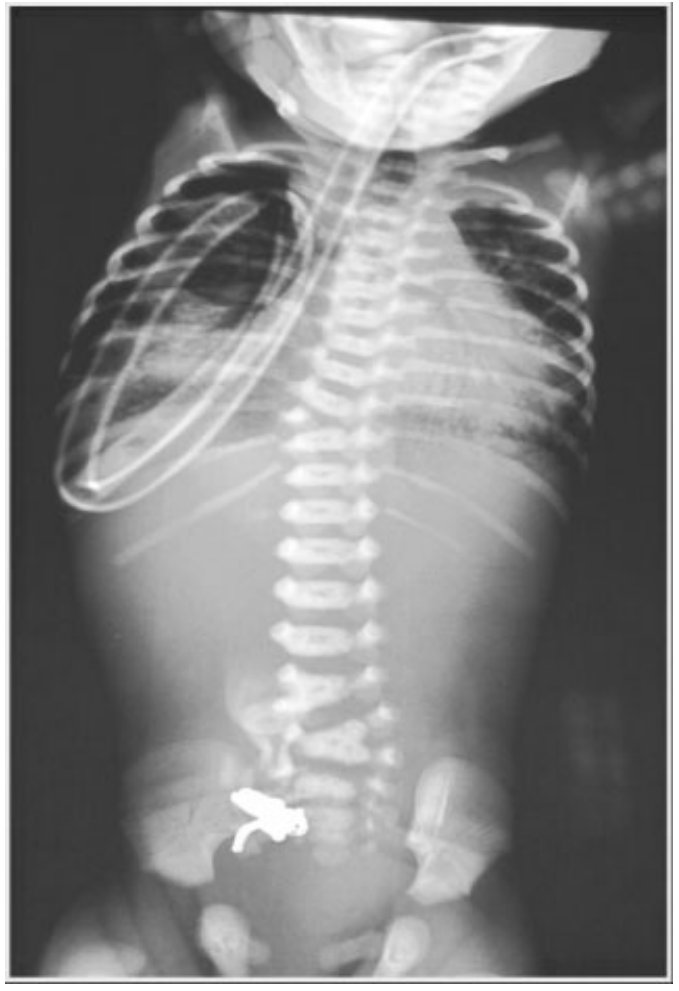

Fig. 1 X-ray showing catheter in right pleural cavity and gasless abdomen.

Infants weighing less than $1,500 \mathrm{~g}$ have weaker pharyngeal muscular structures and are at increased risk of iatrogenic esophageal perforation. ${ }^{1,4}$ Neonatal esophageal perforation can present as pharyngeal or esophageal pseudodiverticulum secondary to a contained cervical perforation (less common), pneumothorax, and perhaps most commonly as esophageal obstruction. This results from compression of the lumen by the mass effect created by the false passage. It is this presentation that can be misdiagnosed as EA. ${ }^{6}$ Unlike atresia, treatment for esophageal perforation typically is medical,

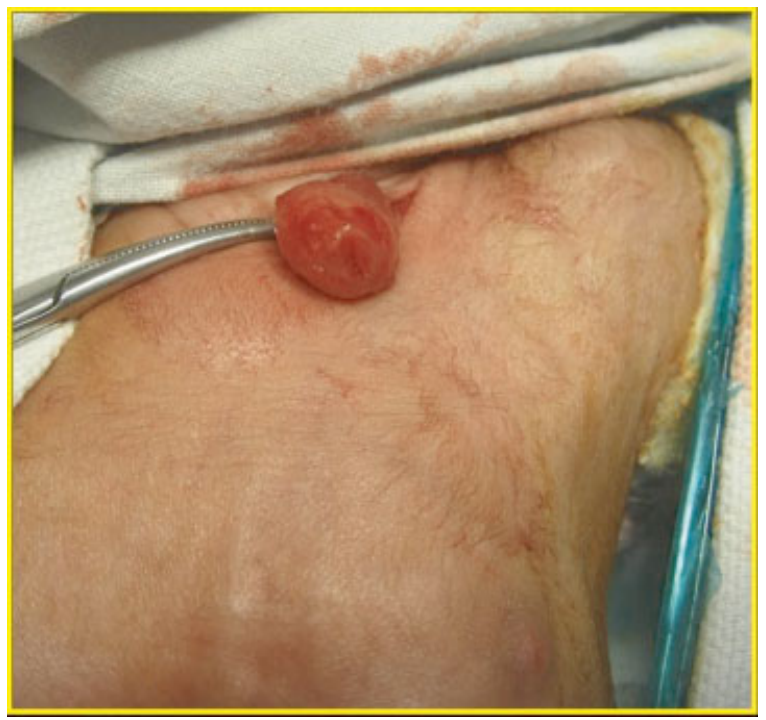

Fig. 2 Intraoperative photograph showing perforation at the tip of upper pouch. with antibiotics, parenteral or gastrostomy feedings, and tube thoracostomy for pneumothorax or pleural effusion., ${ }^{2,6}$ The differentiation of these two entities therefore is very important.

Perforation of upper pouch in EA with TEF (type C) was reported by Wright and Noblett in 1978. They reported it as a complication of using plastic sump catheter for continuous suction of upper pouch. ${ }^{5}$ However, in our case, perforation may have occurred due to the use of stiff catheter for the diagnosis of EA. The use of a stiff catheter can result in one of the following possibilities: (i) uneventful passage into the stomach through a normal esophagus, ruling out EA; (ii) hold up in the upper pouch confirming EA; (iii) iatrogenic perforation of a normal esophagus producing a clinical picture that mimics EA, a false-positive situation; and (iv) iatrogenic perforation of the upper pouch in the case of EA, with the catheter passing into the mediastinum and pleural cavity (as in our case). ${ }^{7}$

Alternatives to the use of stiff catheter for diagnosis of EA are available, such as fluoroscopic swallow study and endoscopy. The risk of aspiration pneumonitis and adequate delineation of upper blind pouch with stiff catheter radiograph makes routine fluoroscopic swallow study less popular. ${ }^{7}$ Bronchoscopy may help in verification of the diagnosis, determination of fistula level, and detection of an occult second fistula with rigid esophagoscopy, which can further clarify the diagnosis and appears safe in experienced hands. ${ }^{8}$ Bronchoscopy and esophagoscopy allow direct visualization of a TEF and blind-ending esophagus. ${ }^{1}$ However, none of these studies are without risk and possibly have greater risk than a catheter radiograph.

\section{Conclusion}

A radiograph after placing catheter (NG) in the upper pouch is an accepted way of diagnosing EA with TEF. The treating surgeon should be vigilant especially in a premature infant or infant weighing less than $1,500 \mathrm{~g}$, as a small force may perforate the upper pouch due to inherent weakness of the dilated tissue and musculature.

\section{Conflict of Interest}

None

\section{References}

1 Knight RB, Webb DE, P Coppola C. Pharyngeal perforation masquerading as esophageal atresia. J Pediatr Surg 2009;44(11): 2216-2218

2 Blair GK, Filler RM, Theodorescu D. Neonatal pharyngoesophageal perforation mimicking esophageal atresia: clues to diagnosis. J Pediatr Surg 1987;22(8):770-774

3 Eklöf O, Löhr G, Okmian L. Submucosal perforation of the esophagus in the neonate. Acta Radiol Diagn (Stockh) 1969;8(2): 187-192

4 Vandenplas Y, Delree M, Bougatef A, Sacre L. Cervical esophageal perforation diagnosed by endoscopy in a premature infant: review of recent literature. J Pediatr Gastroenterol Nutr 1989;8(3): 390-393 
5 Wright VM, Noblett HR. A complication of continuous upper pouch suction in esophageal atresia. J Pediatr Surg 1978;13(4): 369-370

6 Emil SG. Neonatal esophageal perforation. J Pediatr Surg 2004; 39(8):1296-1298
7 Kumar A. Pitfalls in the diagnosis of esophageal atresia. Indian Pediatr 2000;37(11):1287

8 Vandenplas Y, Delree M, Bougatef A, Sacre L. Cervical esophageal perforation diagnosed by endoscopy in a premature infant: review of recent literature. J Pediatr Gastroenterol Nutr 1989;8(3):390-393 